\title{
Hydride Generation in Merging Chamber for Determination of Picogram Amounts of Bismuth by Inductively Coupled Plasma Mass Spectrometry
}

\author{
Tasuku Akagi, Takafumi Hirata and Akimasa Masuda \\ Department of Chemistry, Faculty of Science, The University of Tokyo, Hongo, Tokyo 113, Japan
}

\begin{abstract}
A combination of bismuth hydride generation with a merging introduction technique, where analytes are merged with spray mist from a nebulizer on the way to ICP, has been developed for sensitive determination of bismuth. A sample solution of $20 \mu \mathrm{l}$ was led to a reaction cell inside a merging chamber and was mixed with reductant solution. The generated bismuth tetrahydride was transported to and measured by ICP-MS. With the adoption of flow system, a cyclic procedure of introductions of solutions, reactions and evacuations could be performed sequently with keeping ICP power on. The effects of acidity and volumes of sample solution and reductant on the signal were investigated in detail. The signal intensity was proportional to the amount of bismuth in solution loaded on a reaction cell in the merging chamber. The absolute detection limit of bismuth using reductant of analytical grade was $72 \mathrm{fg}$, which corresponds to $1.8 \mathrm{ppb}$ in $40 \mu \mathrm{l}$ solution. The present method was applied to the solutions of digested standard rocks. Results with good precision and accuracy were obtained.
\end{abstract}

Keywords Inductively coupled plasma-mass spectrometry, bismuth, hydride generation, standard rock

Recently a merging introduction technique has been shown to be an effective introduction method of trace amounts of osmium for inductively coupled plasma source mass spectrometry (ICP-MS). ${ }^{1}$ In the technique, analytes emitted from a solution in a merging chamber are carried to ICP with spray mist from a nebulizer. Since this technique uses wet plasma, a high performance in detection can be obtained after the optimization of operational settings using a standard solution. The efficiency of sample introduction has been much improved compared with a conventional nebulization introduction method, because analytes are not missed into a drain.

Germanium $^{2,3}$, arsenic ${ }^{4,5}$, selenium ${ }^{5,6}$, tin $^{7}$, antimony ${ }^{8,9}$, tellurium ${ }^{5,10}$ and bismuth ${ }^{5,11}$ are hydride-forming elements. Thus hydride generation has been often applied for the separation and the concentration of such elements. ${ }^{2-11}$ The reaction of hydride generation occurs when a sample is mixed with a reductant solution. The present authors have attempted to cause the reaction in the merging chamber, aiming at more effective and more rapid introduction of bismuth into ICP. Furthermore, a flow system is applied to the present study so that the sample solution can be changed easily without the extinction of the plasma.

\section{Experimental}

\section{Apparatus}

The ICP-MS system used was PlasmaQuad type I
(VG Elemental Co. Ltd.). The operating conditions are listed in Table 1. In the merging introduction, nebulization is maintained. Ion lens parameters were optimized with respect to the signal of ${ }^{208} \mathrm{~Pb}$, the solution of which was introduced from a nebulizer. Figure 1 shows the schematic diagram of the present merging introduction system designed for the hydride generation. A peristaltic pump of Gilson type Minipulse 2 was used for a flow system, i.e., for the

Table 1 Instrument operating conditions

\begin{tabular}{ll}
$\begin{array}{l}\text { ICP settings } \\
\text { Incident power }\end{array}$ & $1.35 \mathrm{~kW}$ \\
Reflected power & $<5 \mathrm{~W}$ \\
Argon gas flow rates & \\
$\quad$ Cooling & $131 / \mathrm{min}$ \\
$\quad$ Auxiliary & $1.11 / \mathrm{min}$ \\
$\quad$ Nebulizer & $0.751 / \mathrm{min}$ \\
Nebulizer & \\
Nebulizer type & cross flow \\
Solution up-take rate & $0.6 \mathrm{ml} / \mathrm{min}$ (pumped) \\
Interface & \\
Aperture diameter & $0.9 \mathrm{~mm}$ \\
Skimmer diameter & $1.0 \mathrm{~mm}$ \\
Load coil-aperture spacing & $10 \mathrm{~mm}$ \\
Measurement parameters & \\
Mass fixed at & $209 \mathrm{amu}$ \\
Integration time & $60 \mathrm{~s}$ \\
\hline
\end{tabular}




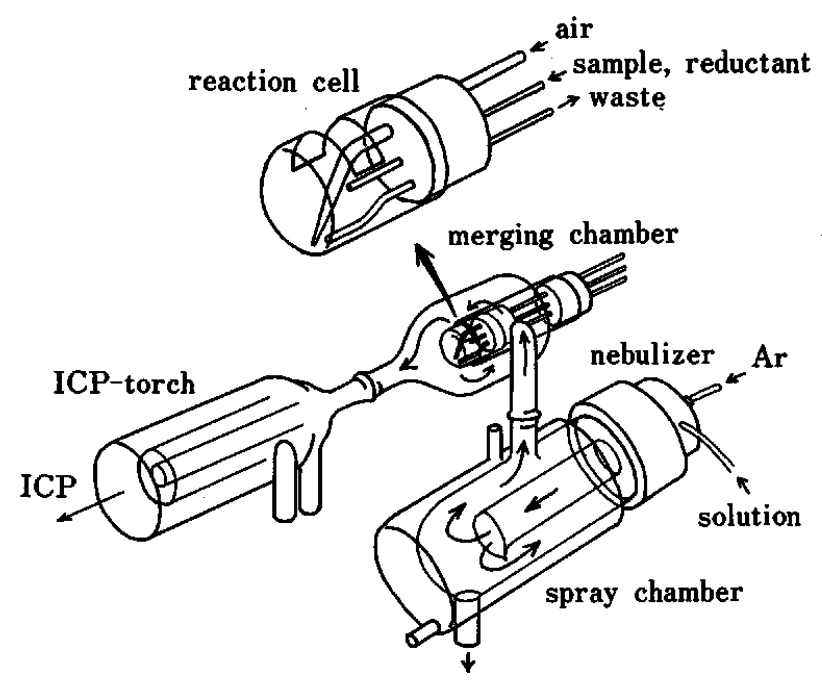

Fig. 1 Schematic diagram of the merging chamber with flow system designed for hydride generation. Reductant and sample are transported into a reaction cell in the merging chamber through the inlet tube. After measurement, the mixture solution is pumped out through the drain tube. To mix the sample and reductant solutions well, air is introduced from the bubbling tube.

introduction and evacuation of solutions.

\section{Chemicals}

All chemicals used were of analytical grade except for hydrochloric acid. Hydrochloric acid of special analytical grade purchased from Wako Chemicals was used. All the chemicals were used without further purification. Three per cent sodium borohydride solutions were prepared by dissolving the reagent of Wako Chemicals with pure water. To stabilize the reductant, $0.3 \mathrm{ml}$ of $2 \mathrm{M}$ sodium hydroxide was added to $100 \mathrm{ml}$ of the sodium borohydride solution.

\section{Samples}

Powder samples (each about $1 \mathrm{~g}$ ) of standard rocks, JB-1 and JGb-1, distributed by Geological Survey of Japan were digested with $15 \mathrm{ml}$ of hydrofluoric and $5 \mathrm{ml}$ of perchloric acids. They were dissolved in $3 \mathrm{M}$ hydrochloric acid.

\section{Recommended procedure}

The ion-lens parameters were first optimized by introducing a $\mathrm{Pb}$ standard solution through a nebulizer. Then $10 \mu \mathrm{l}$ of $3 \%$ sodium borohydride solution was pumped into the reaction cell in the merging chamber. After $10 \mathrm{~s}, 40 \mu \mathrm{l}$ of sample or standard solution in $3 \mathrm{M}$ hydrochloric acid were pumped to the reaction cell. They were mixed together in the reaction cell and bismuth tetrahydride was generated (During the whole procedure, air was always bubbled into the reaction cell through the bubbling tube, to mix well the sample and the reductant.). The gaseous hydride was mixed with spray mist from a nebulizer in the merging

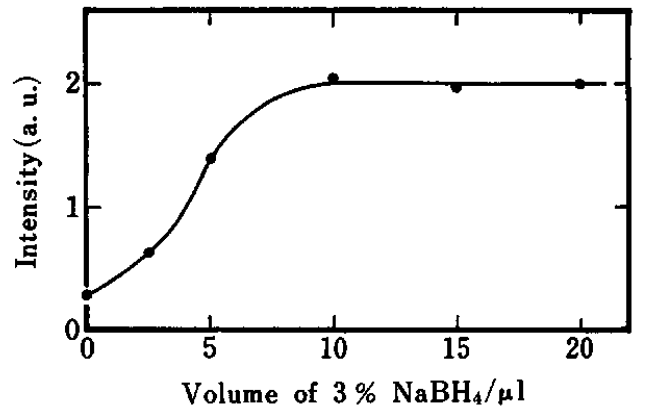

Fig. 2 Effect of amount of $3 \%$ sodium borohydride solution on the detected signal counts. Sample volume was $40 \mu l$ and acid concentration was $\mathrm{HCl} 3 \mathrm{M}$.

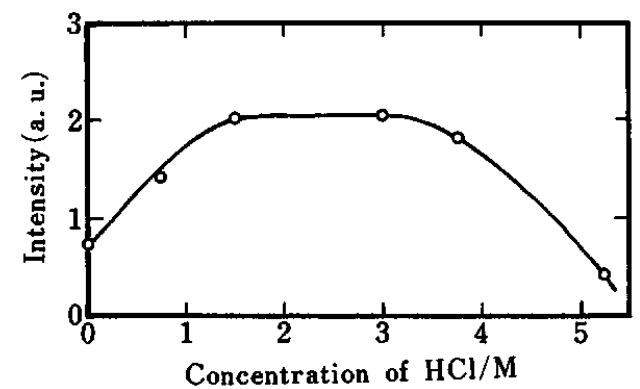

Fig. 3 Effect of acidity on the detected signal counts. Sample volume was $40 \mu \mathrm{l}$ and $10 \mu \mathrm{l}$ of $3 \%$ sodium borohydride solution was added.

chamber and carried to ICP. After measurement for $2 \mathrm{~min}$, the mixture was pumped out to the drain before the analysis of the next sample.

\section{Results and Discussion}

\section{Optimization of hydride generation}

The amounts of reductant and acid affect the reaction of hydride generation. In Figs. 2 and 3, the effects of sodium borohydride and acidity on the signal counts of bismuth are shown. In this experiment, the volume of sample loaded on the reaction cell is $40 \mu \mathrm{l}$ each time. As can be seen in Fig. 2, the signal counts increase with the volume of $3 \%$ sodium borohydride solution and reach a plateau when the volume is larger than $10 \mu \mathrm{l}$. Figure 3 shows that the acid concentrations between 1.5 and $3 \mathrm{M}$ give the highest signal counts. For the optimized conditions for the reaction, we have chosen $10 \mu \mathrm{l}$ as a volume of $3 \%$ sodium borohydride solution and $3 \mathrm{M}$ as a concentration of hydrochloric acid.

\section{Volume of sample}

No significant change in the signal counts of bismuth was observed when the volume was changed from 10 to $60 \mu \mathrm{l}$. In the case where the sample volume is 
small $(10 \mu \mathrm{l})$, bubbling of air sometimes moved reductant aside so that the sample solution could not be mixed with the reductant. A larger volume is more favorable for the sample solutions containing a lower concentration of bismuth. The maximum volume of sample loadable on the reaction cell is $60 \mu \mathrm{l}$ and the bubbling sometimes caused overflow of the sample from the reaction cell when the volume was $60 \mu \mathrm{l}$. Therefore, $40 \mu \mathrm{l}$ was chosen as the volume of sample for further study.

\section{Typical signal profile}

Using the recommended procedure and the optimized conditions of the reaction, we obtained the signal profile at ${ }^{209} \mathrm{Bi}$ shown in Fig. 4 . The signal intensity rapidly increased when the sample was mixed with the reductant. After reaching the maximum, it decreased gradually. More than $95 \%$ of bismuth was detected during the first $60 \mathrm{~s}$. Thus the signal counts were integrated for $60 \mathrm{~s}$.

\section{Calibration curve and detection limit}

The present flow system enables us to change the

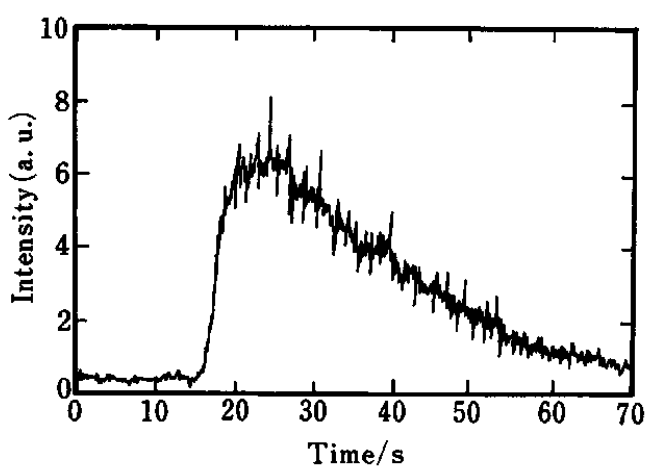

Fig. 4 Typical signal profile at ${ }^{209} \mathrm{Bi}$. Sample was $40 \mu \mathrm{l}$ in $3 \mathrm{M}$ $\mathrm{HCl}$ and $10 \mu \mathrm{l}$ of $3 \%$ sodium borohydride solution was added.

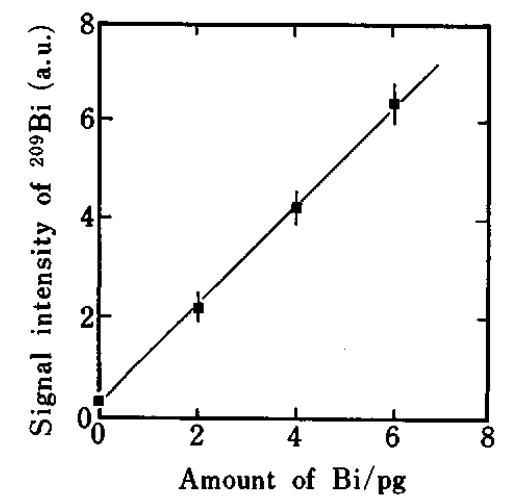

Fig. 5 Calibration curve of ${ }^{209} \mathrm{Bi}$ obtained by the present merging chamber/hydride generation method. Sample was $40 \mu \mathrm{l}$ in $3 \mathrm{M} \mathrm{HCl}$ and $10 \mu \mathrm{l}$ of $3 \%$ sodium borohydride solution was added. Integration time of signal was $60 \mathrm{~s}$. sample without the extinction of ICP. Therefore a calibration curve can be made by loading several solutions containing different amounts of bismuth. In Fig. 5, the calibration curve is shown. A straight line was obtained for the amount of bismuth from 0 to $8 \mathrm{pg}$. Since the present system is very sensitive, larger amounts of bismuth were not tried to avoid the memory effect. No concern about the memory effect was necessary when the amount of bismuth loaded was less than $10 \mathrm{pg}$ (In order to eliminate the possible memory effect, it is advisable to wash the reaction cell with sodium borohydride and hydrochloric acid after each measurement). The sensitivity, blank counts and detection limit of the present method are summarized in Table 2. The detection limit is the concentration of bismuth corresponding to three times deviation of blank counts $(3 \sigma)$. The blank experiments gave the average signal counts of 3235 with a standard deviation of 729. Therefore the absolute detection limit of the present method is about $72 \mathrm{fg}$. It should be noted that in the present method, no reagents purified specially were used. We expect that the use of the purified reagents of sodium borohydride and hydrochloric acid can lower the blank counts and detection limit.

\section{Application to rock sample}

The present method was applied to the digested samples of two standard rocks, JB-1 and JGb-1. The analytical results are summarized in Table 3 along with the certified values. ${ }^{12}$ Forty microliters of the digested sample was loaded on the reaction cell. The analytical results for both the rock samples agreed well with the certified values. It should be emphasized that the two standard rocks are of different types and have different matrix compositions. Even for the samples of the different matrix compositions, the present method

Table 2 Sensitivity, blank counts and detection limit

\begin{tabular}{lc}
\hline Sensitivity & 10080 counts $/ \mathrm{pg}$ \\
Blank counts $^{\mathrm{a}}$ & $3235 \pm 729$ counts \\
Detection limit & \\
absolute & $72 \mathrm{fg}$ \\
in $40 \mu \mathrm{l}$ solution & $1.8 \mathrm{pg} / \mathrm{g}$ \\
\hline
\end{tabular}

a. $n=7$. The error is 3 times standard deviation. b. Calculated as $3 \sigma$ of blank counts.

Table 3 Analytical results of standard rocks

\begin{tabular}{|c|c|c|}
\hline & \multicolumn{2}{|c|}{ Concentration of bismuth $/ \mathrm{ng} \mathrm{g}^{-}$} \\
\hline & This work $^{\mathrm{a}}$ & Ref. 12 \\
\hline $\mathrm{JB}-1^{\mathrm{b}}$ & $33.2 \pm 1.4$ & 31 \\
\hline $\mathbf{J G b}-1^{\mathrm{b}}$ & $13.0 \pm 0.6$ & 14 \\
\hline
\end{tabular}

a. Number of analysis is 3. Errors are $1 \sigma$. b. Dilution factor of digested solution is $\mathbf{5 0 0}$ for JB-1 and 200 for JGb-1. Forty microliters of the digested solutions were loaded on the reaction cell. 
could be applied to the digested solution without chemical separation.

In conclusion, the merging introduction technique has been studied as a new sensitive introduction method for ICP-MS analysis. It has been shown to be effective for the sensitive analysis of osmium. ${ }^{1}$ With a merging chamber designed for the hydride generation method and with adoption of flow system, the merging introduction is found to be very effective for the hydride generation method, too. The absolute detection limit of bismuth was about $72 \mathrm{fg}$. It shoulld be noted that the present system can be applied to the analyses of the other hydride generating elements such as arsenic, tellurium, selenium, etc. with some modifications of reaction conditions. Since the merging introduction method makes it possible to perform very sensitive analyses with simple procedures, it is strongly recommended for the trace analysis in a variety of fields using not only ICP-MS but also ICP-AES.

The work was supported in part by a Grant-in-Aid for Fundamental Scientific Research from the Ministry of Education, Science and Culture, Japan.

\section{References}

1. T. Hirata, T. Akagi, H. Shimizu and A. Masuda, Anal. Chem., 61, 2263 (1989).

2. M. O. Andreae and P. N. Froelich, Jr., Anal. Chem., 53, 287 (1981).

3. G. A. Hambrick III, P. N. Froelich, Jr., M. O. Andreae and B. L. Lewis, Anal. Chem., 56, 421 (1984).

4. M. Yamamoto, K. Fujishige, H. Tsubota and Y. Yamamoto., Anal. Sci., 1, 47 (1985).

5. M. Yamamoto, M. Yasuda and Y. Yamamoto, Anal. Chem., 57, 1382 (1985).

6. S. N. Willie, R. E. Sturgeon and S. S. Berman, Anal. Chem., 58, 1140 (1986).

7. O. F. X. Donard, S. Rapsomanikis and J. H. Weber, Anal. Chem., 58, 772 (1986).

8. M. O. Andreae, J.-F. Asmode, P. Foster and L. Van't Dack, Anal. Chem., 53, 1766 (1981).

9. R. E. Sturgeon, S. N. Willie and S. S. Berman, Anal. Chem., 57, 2311 (1985).

10. M. O. Andreae, Anal. Chem., 56, 2064 (1984).

11. D. S. Lee, Anal Chem., 54, 1682 (1982).

12. K. Govindaraju, Geostandards Newslett., 1984, vol. VIII, Special Issue. 